\title{
Taming the beast: rabies control in the cradle of mankind
}

\author{
Gianluca D’Amico ${ }^{1}$, Andrei D. Mihalca ${ }^{1}$, Cristian Domşa ${ }^{1}$, Kateřina Albrechtová ${ }^{2}$, Attila D. \\ Sándor ${ }^{1}$, David Modrý ${ }^{3,4,5}$ \\ ${ }^{1}$ Department of Parasitology and Parasitic Diseases, University of Agricultural Sciences and Veterinary Medicine \\ Cluj-Napoca, Calea Mănăştur 3-5, Cluj-Napoca, 400372, Romania; ${ }^{2}$ Department of Biology and Wildlife Diseases, \\ Faculty of Veterinary Hygiene and Ecology, University of Veterinary and Pharmaceutical Sciences, Palackého 1-3, \\ 61242 Brno, Czech Republic; ${ }^{3}$ Biology Center, Institute of Parasitology, Academy of Sciences of the Czech Republic, \\ Branišovská 31, 37005 České Budějovice, Czech Republic; ${ }^{4}$ CEITEC - Central European Institute of Technology, \\ University of Veterinary and Pharmaceutical Sciences, Brno, Czech Republic; ${ }^{5}$ Department of Parasitology, \\ University of Veterinary and Pharmaceutical Sciences, Palackého 1-3, 61242 Brno, Czech Republic
}

\begin{abstract}
Between 2006 and 2012, a rabies control programme has been conducted in the area of Lake Turkana in northern Kenya. Spatial data obtained for this project were analysed with the aim of assessing the importance of dog home ranges with the view of possible overlapping between dog populations from adjacent localities. In contrast to our expectation of the maximum home ranges of dogs in the harsh semi-desert environment, the results provided by geographical information system (GIS) analysis showed that in 14 out of 16 localities considered for the study, the dog populations were fully isolated from each other. The data obtained should be helpful for designing rabies control strategies.
\end{abstract}

Keywords: dogs, rabies, geographical information systems, Kenya.

Link: https://www.youtube.com/watch?v=qhtZkWrFnik\&feature=em-upload_owner

\section{Background}

Rabies is a deadly viral zoonotic disease with dogs as the major source for human infection. It is estimated to cause more than 55,000 human deaths per year but its social impact is even more critical as between $35-50 \%$ of the victims are children (Briggs and Mahendra, 2007). The vast majority of human rabies infection takes place in tropical countries in Asia and Africa. Knobel et al. (2005) estimated that the overall incidence of human rabies in Africa is between 2.0 and 3.6 persons per 100,000 inhabitants. Unlike European countries, where wildlife (i.e. foxes) play a major role (Mulatti et al., 2011), infected dogs constitute the main risk for humans in Kenya and other African countries. Hence, it has been advocated that the control measures should include mass vaccination of these animals (Cleaveland et al., 2003). It has been shown

\footnotetext{
Corresponding author: Andrei D. Mihalca

Department of Parasitology and Parasitic Diseases

University of Agricultural Sciences and

Veterinary Medicine Cluj-Napoca

Calea Mǎnǎștur 3-5, Cluj-Napoca, 400372, Romania

Tel. +40 264596 384; Fax +40 264593792

E-mail: amihalca@usamvcluj.ro
}

that single-shot vaccination with inactivated vaccine Rabisin ${ }^{\circledR}$, Merial (Kayali et al., 2003) of $70 \%$ of the dog population is sufficient to prevent outbreaks of canine rabies leading to interruption of human exposure for 6 years (Zinsstag et al., 2009). However, due to various factors (i.e. high cost, inadequate infrastructure for public health and veterinary services, cultural objections, etc.), these methods are difficult to implement in developing countries (Cleaveland et al., 2003). Thus the proportion of vaccinated dogs in lowresource countries usually falls way below the recommended thresholds. Another important factor influencing the success of vaccination programmes is the campaign frequency, mainly in areas with a high turnover rate of the dog populations (Cleaveland et al., 2001). Apart from dog vaccination, two other components should be considered for a successful rabies control: public awareness and availability of post-exposure prophylaxis (PEP).

Between 2006 and 2012, a team of European veterinarians, in collaboration with local authorities, have conducted a rabies control in the area of Lake Turkana in northern Kenya, a place famous mainly for its abundance in fossils of early hominids. The region is still inhabited by semi-nomadic tribes depending on domestic animals and it is known as an area with high human incidence of rabies. This programme focused 
on three components: mass vaccination of dogs, public awareness and PEP. During annual campaigns, the total number of vaccinated dogs was 1,472 in 16 localities, while the number of people receiving PEP in two hospitals was around 100. Based on the estimation of the dog population we have calculated the percentage of vaccinated dogs in each locality. The dog population was calculated based on data of the 2009 human census (http://www.knbs.or.ke/surveys.php) and an estimated dog/human ratio of 1:8 (Kitala et al., 2001). Moreover, in each locality, we have calculated the mean age (in months) of the vaccinated dogs.

\section{Study area}

The study area is in northern Kenya, south-east of the Rift Valley near Lake Turkana, located between Latitude $2.00^{\circ} \mathrm{N}$ and $3.00^{\circ} \mathrm{N}$ and Longitude $36.42^{\circ} \mathrm{E}$ and $37.09^{\circ} \mathrm{E}$. Most of the area is sparsely populated semi-desert, with two forested mountains (Mount Kulal and Mount Ng'iro).

In order to achieve a proper percentage of vaccinated dogs from the total estimated populations in each locality, we used a new geographical information systems (GIS) approach considering the range of dogs and the possible interconnectivity of dog populations from adjacent villages. This is important mainly in order to improve the success of future rabies control programmes through targeted and more cost-effective campaigns in remote and arid areas.

The basic assumption in dealing with spatial data was the maximum range of dogs in the harsh semidesert environment. In such conditions, the maximum expansion of free range outside the inhabited area is about $1 \mathrm{~km}$. Given the spatial distribution of villages in semi-desert, northern Kenya, the dog populations are isolated from each other, and the exchange between such populations is mediated by humans only.

The first step in mapping the ranges of dogs was to identify the human settlements in the working area. We took global positioning system (GPS) coordinates at each village using a Garmin Dakota 10 instrument. As dogs are associated with humans, the range of the local dog population was considered the maximum extent of the settlement. To map the external boundaries we used the satellite imagery provided by Google Earth, whose high-resolution, spatial imagery provided us with the appropriate tool to identify the peripheral components of each village (mostly cattle and sheep enclosures accompanied by traditional huts). Hence, we obtained a maximum extent polygon for each village, to be considered as the maximum inhabited area by local human population. The next step was to calculate the maximum free moving range for the local dog populations in each village. Starting from the digitised village polygons, we used the buffer tool with $1,000 \mathrm{~m}$ distance to calculate the maximum range that the dogs can travel outside the inhabited areas. For calculating the buffers of $1,000 \mathrm{~m}$ around each inhabited area, we used quantum GIS (QGIS) open-source software (Buffer/Geoprocessings tools), having set the resulting overlapping buffers to merge and dissolve. Hence, we obtained a maximum free moving range for local dog population around each inhabited area.

Village polygons with boundaries closer than the set $1,000 \mathrm{~m}$ distance have merged in single continuous buffers. In terms of dog population, such cases were henceforth considered as single units and all subsequent data refer to buffer as a whole, rather than to each component village as separate.

In order to assess the possible impact of climate on some dog population parameters, we considered climatic data from the Worldclim spatial database (http://www.worldclim.org). Four different climatic parameters were employed in order to verify the possible correlations with the dog data: the annual mean temperature, the maximum temperature of the warmest month, the minimum temperature of the coldest month and the amount of annual precipitation. For each of the obtained buffers, climatic data for all four parameters were extracted using the System for Automated Geoscientific Analyses (SAGA). The climatic data grid cell values were used to calculate minimum, maximum, mean and standard deviation in each buffer.

The meta-analyses of the vaccination data showed a high heterogeneity of the percentage of vaccinated dogs in various localities. The results provided by the GIS analysis showed that in 14 out of the 16 localities, the dog population were fully isolated from each other. Hence, we have merged the dog data in these locations, resulting in a final number of 14 isolated dog populations. Our approach shows this new parameter (interconnectivity of dog populations in adjacent localities) should be considered when implementing rabies control programmes in order to correctly evaluate the percentage of vaccinated dogs. Moreover, our results suggest that when implementing rabies control programmes, all its components (vaccination of dogs, public awareness and PEP) should be performed per group of localities if there is a clear indication of dog range overlapping. 
The mean age of vaccinated dogs was variable in each locality, ranging from 5.25 to 63.37 months. No correlation was found between the mean age and the climatic data. However, the high turn-over rate of dog populations in harsh environments like northern Kenya suggests the need of sustained, bi-annual vaccination campaigns.

\section{Outlook}

When implementing rabies control programmes, all adjacent localities where the home range of the dogs overlaps must be included. Geospatial tools are effective methods for estimating the real field situation concerning the dog population and home-range overlap.

\section{Box 1. Overall aim.}

The general purpose of this report is to highlight the importance of geospatial tools for the successful implementation of rabies control programmes. The overall aim was to assess the importance of the dog home range, with the view of possible overlapping between dog populations from adjacent localities and its implementation into control strategies.

Box 2. Applied software.

- Google Earth. Free high resolution satellite imagery and global coverage make this software an extremely valuable tool in dealing with spatial data. Several different image sources, such as DigitalGlobe and Cnes/SPOT Images compiled under Google Earth umbrella, provided us with an easy-to-use tool in digitizing the extent of the settlements.

- QGIS. Free, fully fledged GIS software was used both to digitise the settlement polygons and to calculate the buffers. Google satellite imagery is accessible for use in this software via plug-ins. First, using satellite imagery we checked and corrected the digitised polygons, in order to ensure full coverage of the settlements. Second, we used the buffer tool (geoprocessing) to calculate the required range and to merge the ones closer than the set limit (1000 m).

- SAGA. A useful GIS software, oriented mainly towards spatial analysis, provides a wide range of spatial analysis tools, especially for raster and grid data. This software was used to extract the grid cell values in case of climatic data. First, the previously obtained buffers were used to clip the climatic grids (clip grid with polygon tool). Second, for the obtained buffers the required values were calculated for each climatic data set separately (grid statistics for polygon tool).

\section{Acknowledgement}

We are indebted to all the people involved in the project during all study years: veterinarians, students, volunteers, local helpers, local communities, elders' council, etc. Mount Kulal Rabies Control is supervised by VSF (Vétérinaires Sans
Frontières) Czech Republic. The entire vaccination project and on-going research would not be possible without generous support from many different donors (for entire list see www.vsfcz.eu), State Veterinary Institute Prague (Czech Republic), University of Agricultural Sciences and Veterinary Medicine (Cluj-Napoca, Romania) and Institute of Parasitology, Biology Center ASCR (České Budějovice, Czech Republic). Part of the field work was financed by the Internal Grant Agency of University of Veterinary and Pharmaceutical Sciences (UPVS), Brno: UPVS IGA 55/2010/FVL. We are pleased to acknowledge the support by District Veterinary Offices of Marsabit and Laisamis, Kenya, the Embassy of the Czech Republic in Nairobi, including Mrs. Margita Fuchsová, Czech Ambassador to Kenya.

\section{References}

Briggs DJ, Mahendra BJ, 2007. Public health management of humans at risk. In: Rabies: scientific basis of the disease and its management. Wunner WHM, Jackson AC (eds). Elsevier, 545-572 pp.

Cleaveland S, Kaare M, Tiringa P, Mlengeya T, 2001. A dog rabies vaccination campaign in rural Africa: impact on the incidence of animal rabies and human bite injuries. Proceedings of the Southern and Eastern African Rabies Group. World Health Organization Meeting in Lilongwe, Malawi, pp. 114-126.

Cleaveland S, Kaare M, Tiringa P, Mlengeya T, Barrat J, 2003. A dog rabies vaccination campaign in rural Africa: impact on the incidence of dog rabies and human dog-bite injuries. Vaccine 21, 1965-1973.

Kayali U, Mindekem R, Yémadji N, Vounatsou P, Kaninga Y, Ndoutamia AG, Zinsstag J, 2003. Coverage of pilot parenteral vaccination campaign against canine rabies in N'Djamena, Chad. Bull World Health Organ 81, 739-744.

Kitala A, McDermott J, Kyule M, Gathuma J, Perry B, Wandeler A, 2001. Dog ecology and demography information to support the planning of rabies control in Machakos district, Kenya. Acta Trop 78, 217-230.

Knobel DL, Cleaveland S, Coleman PG, Fèvre EM, Meltzer MI, Miranda MEG, Shaw A, Zinsstag J, Meslin FX, 2005. Reevaluating the burden of rabies in Africa and Asia. Bull World Health Organ 83, 360-368.

Mulatti P, Ferrè N, Patregnani T, Bonfanti L, Marangon S, 2011. Geographical information systems in the management of the 2009-2010 emergency oral anti-rabies vaccination of foxes in north-eastern Italy. Geospat Health 5, 217-226.

Zinsstag J, Dürr S, Penny MA, Mindekem R, Roth F, Menendez Gonzalez S, Naissengar S, Hattendorf J, 2009. Transmission dynamics and economics of rabies control in dogs and humans in an African city. Proc Nat Acad Sci USA 106, 14996-15001. 